\title{
DISEÑO DE INSTRUMENTOS ECONÓMICOS PARA LA GESTIÓN SOSTENIBLE DE ESPACIOS NATURALES: UN ENFOQUE A TRAVÉS DE LA HETEROGENEIDAD SOCIAL
}

\author{
$\underline{\text { José A. Zabala }^{a^{*}}}$, José A. Albaladejo-Garcíab \\ a Departamento de Economía de la Empresa. Universidad Politécnica de Cartagena. (Cartagena, \\ joseangel.zabala@upct.es \\ ${ }^{b}$ Departamento de Economía Aplicada. Universidad de Murcia. (Murcia: joseantonio.albaladejo@um.es)
}

\section{Resumen}

Los instrumentos económicos ayudan al diseño de estrategias de gestión sostenible de espacios naturales. Para su aplicación, es crucial conocer el valor económico que la sociedad otorga a dichos espacios, así como los factores sociales que lo determinan. Este trabajo tuvo como objetivo diseñar instrumentos económicos para preservar un espacio incluido en la Red Natura 2000 a partir de la valoración y comprensión de los factores sociales que motivan la disposición a pagar por medidas de gestión sostenibles. Para ello se utilizó un ejercicio de valoración contingente, con la población local de los espacios protegidos del Cabezo de la Jara y Rambla de Nogalte (Región de Murcia, España). La heterogeneidad fue analizada a través de un modelo de clases latentes.

Los resultados pusieron de relieve la heterogeneidad social existente, determinada por el nivel de ingresos familiares y las actitudes ambientales. Se proponen así dos instrumentos económicos, impuestos directos ambientales y tarifas de usuario, con el fin de asegurar el apoyo financiero a las medidas y una amplia aceptación social. Ello permitirá guiar a los gestores públicos en la formulación de políticas de conservación de la naturaleza en aras de maximizar su aceptabilidad.

Palabras clave: Aceptabilidad social, Diseño de políticas, Disposición a pagar, Valoración contingente, Valoración de no-mercado.

\section{Introducción y objetivos}

La valoración de los beneficios que proporciona los espacios naturales y su integración en los mecanismos de formulación de políticas puede ser una forma efectiva para evidenciar la importancia de la gestión sostenible de estos espacios [Ives y Kendal (2014)]. Sin embargo, conocer el valor que la sociedad otorga a los planes de gestión ambiental no es suficiente para asegurar su sostenibilidad. Necesitamos ir más allá y evaluar y comprender los factores que determinan este apoyo social.

Asimismo, es necesario lograr la aceptabilidad social de las medidas de gestión para la conservación de estos espacios a fin de garantizar su éxito y perdurabilidad a largo plazo. Por tanto, el diseño de instrumentos económicos, basado en la evaluación ex ante de la heterogeneidad de las preferencias, sirve para orientar a los responsables políticos en su compromiso de implementar aquellos incentivos que aseguren su aceptabilidad [Rode et al. (2016)].

En este contexto, este trabajo tiene como objetivo evaluar los factores que determinan la demanda social y el valor de medidas de gestión sostenible para la conservación de los espacios naturales y propone instrumentos económicos para su apoyo financiero, buscando asegurar su aceptabilidad social.

\section{Metodología}

Como caso de estudio se seleccionaron los espacios protegidos de Cabezo de la Jara y Rambla de Nogalte (en adelante, ENP) (Región de Murcia, España), que pertenecen a la Red Natura 2000. Este espacio natural está conformado por un monte calcáreo, el cual tiene una altitud máxima de 1,247 m, y está cubierto por una vegetación de tipo matorral y por comunidades abiertas de encina y pino carrasco; y una rambla típica mediterránea de régimen torrencial. Para garantizar su conservación, se propusieron una serie de medidas de gestión sostenible, seleccionadas en base a las tres esferas de la sostenibilidad, ambiental, económica y social.

El valor asociado a la gestión sostenible de este espacio natural se determinó mediante el método de valoración contingente. Se preguntó a la población si estaría dispuesta a pagar (DAP) por apoyar medidas de gestión sostenible para la conservación del ENP. La pregunta de valoración se llevó a cabo mediante un formato mixto, que combinaba una pregunta dicotómica con una en formato abierto, donde los encuestados indicaban su DAP máxima. El vehículo de pago fue una reasignación de los impuestos que actualmente paga cada hogar para destinarlos a apoyar la implementación de estas medidas durante los próximos cinco años. El ejercicio de valoración contingente fue implementado con el apoyo de un cuestionario, donde además se incluían cuestiones para abordar los vínculos entre los encuestados y el ENP, la percepción social 
sobre el estado de conservación y las medidas a implementar, así como preguntas de índole socioeconómico y actitudinal para caracterizar a los encuestados y servir de base para el posterior análisis de heterogeneidad. Las variables actitudinales cuantificaban el grado de compromiso ambiental a través de tres índices (ICA): compromiso afectivo (sentimientos hacia los problemas ambientales), compromiso verbal (disposición a actuar en relación con estos problemas) y compromiso real (acciones reales tomadas para abordar estos problemas) [Maloney et al. (1975)].

Los resultados fueron analizados mediante un modelo Tobit con el fin de conocer los factores sociodemográficos y actitudinales que determinan la DAP máxima. Una vez determinados dichos factores, estos resultados se emplearon para desarrollar un análisis de clases latentes. Dicho análisis se utilizó para identificar la existencia de distintas clases de encuestados en función de los factores identificados, lo que ayuda a comprender cómo se distribuye la DAP en la muestra. De este modo, las clases resultantes fueron empleadas de partida para el diseño de instrumentos económicos de apoyo a la gestión sostenible del ENP.

La población objetivo fueron los 85.270 hogares de la comarca del Valle del Guadalentín, donde se ubica el espacio natural. Los datos fueron tomados en noviembre de 2015 a través de encuestas personales, obteniéndose una muestra final de 150 hogares, lo cual proporciona, a un nivel de confianza del $95 \%$ para una variable dicotómica, un error muestral del 8 y 4,8\% para proporciones intermedias y extremas, respectivamente.

\section{Resultados}

\subsection{Valoración económica y clases latentes}

Los resultados de la valoración contingente revelaron que, entre los 150 encuestados, 113 mostraron una DAP positiva, mientras que 26 fueron identificados como protestas, los cuales fueron excluidos del análisis posterior. En promedio, la población local estaba dispuesta a reasignar 19,35€/hogar/año de los impuestos que actualmente paga para apoyar la gestión sostenible del ENP. La agregación de este valor sobre todos los hogares del Valle del Guadalentín indica que los beneficios de su conservación tienen un valor económico total (VET) de 1,65 M€ anuales.

Cuadro 1. Modelo Tobit. Factores que explican la DAP máxima

\begin{tabular}{|c|c|c|}
\hline & Coef. (Err. Est.) & \\
\hline Constante & $-49.87(20.06)$ & $* * *$ \\
\hline \multicolumn{3}{|c|}{ Factores sociodemográficos } \\
\hline Género (mujer) & $-3.05(3.52)$ & \\
\hline Edad & $0.13(0.18)$ & \\
\hline Renta anual & $3.02 \cdot 10^{-4}\left(1.77 \cdot 10^{-4}\right)$ & $*$ \\
\hline Tamaño hogar & $-0.97(1.48)$ & \\
\hline Ed. Primaria ${ }^{a}$ & $4.57(9.65)$ & \\
\hline Ed. secundaria ${ }^{a}$ & $5.01(9.73)$ & \\
\hline Ed. superior ${ }^{\mathrm{a}}$ & $5.43(10.31)$ & \\
\hline Jubilado $^{\mathrm{b}}$ & $-3.20(10.00)$ & \\
\hline Estudiante $^{\mathrm{b}}$ & $8.32(13.78)$ & \\
\hline Amo de casa ${ }^{b}$ & $2.18(7.89)$ & \\
\hline Trabajador activo ${ }^{b}$ & $0.39(6.47)$ & \\
\hline \multicolumn{3}{|c|}{ Factores actitudinales } \\
\hline ICA-Afectivo & $10.50(3.52)$ & $* * *$ \\
\hline ICA-Verbal & $-0.29(1.63)$ & \\
\hline ICA-Real & $2.21(1.34)$ & $*$ \\
\hline \multicolumn{3}{|l|}{ Relación con el ENP } \\
\hline Usuario & $2.51(3.36)$ & \\
\hline
\end{tabular}

Nota: Nivel de significatividad al $* 10 \%, * * * 1 \%$.

${ }^{a}$ Nivel de referencia: Sin estudios

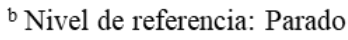

Fuente: Elaboración propia

Los factores que explican la DAP máxima fueron analizados mediante un modelo Tobit, tal como recoge el Cuadro 1. Respecto las variables sociodemográficas, solo la renta del hogar resultó significativa para determinar el valor económico que las personas otorgan a la gestión sostenible del ENP. En cuanto a las variables actitudinales, los elementos afectivos y reales del compromiso ambiental resultaron significativos. 
Cuanto mayor es la preocupación medioambiental de los encuestados, mayor es su DAP, ya que ambos coeficientes muestran signos positivos.

La renta y el compromiso ambiental se emplearon como clústeres para establecer clases latentes. El número de clases latentes en cada clúster se determinó utilizando los criterios AIC y BIC, los cuales mostraron que existían 2 y 4 clases de acuerdo a su renta y compromiso ambiental, respectivamente. En el Cuadro 2 se muestran los resultados de este análisis. En cuanto al clúster de renta, la clase 1 comprende el $72 \%$ de los encuestados (rentas bajas y medias, con una renta media anual de $16.900 €$ ), mientras que la clase 2 incluye el 28\% de los encuestados, aquellos que tienen mayores ingresos anuales, con una media de unos $36.000 €$. En el clúster de compromiso ambiental, los encuestados se agruparon en 4 clases, en orden ascendente según su compromiso ambiental afectivo y real.

Cuadro 2. Análisis de clases latentes

\begin{tabular}{|c|c|c|c|c|c|c|}
\hline & \multicolumn{2}{|l|}{$\begin{array}{c}\text { Clase } 1 \\
\text { Coef. (Err. Est.) }\end{array}$} & \multicolumn{2}{|l|}{$\begin{array}{c}\text { Clase } 2 \\
\text { Coef.(Err. Est.) } \\
\end{array}$} & $\begin{array}{c}\text { Clase } 3 \\
\text { Coef. (Err. Est.) } \\
\end{array}$ & $\begin{array}{c}\text { Clase } 4 \\
\text { Coef.(Err. Est.) }\end{array}$ \\
\hline \multicolumn{7}{|c|}{ Clúster de renta } \\
\hline \multicolumn{7}{|l|}{ Media } \\
\hline Renta anual & $16.898,03(706,25)$ & $* * *$ & $36.088,57(1.293,20)$ & $* * *$ & & \\
\hline \multicolumn{7}{|c|}{ Asignación de clases } \\
\hline Prob. (\%) & $71,80(4,40)$ & & $28,20(4,40)$ & & & \\
\hline Constante & & & $-0,93(0,22)$ & $* * *$ & & \\
\hline \multicolumn{7}{|c|}{ Clúster de compromiso ambiental } \\
\hline \multicolumn{7}{|l|}{ Media } \\
\hline ICA-Afectivo & $3,72(0,06)$ & $* * *$ & $4,88(0,04)$ & $* * *$ & $4,89(0,05) * * *$ & $4,94(0,06) * * *$ \\
\hline ICA-Real & $1,83(0,12)$ & $* * *$ & $1,28(0,10)$ & $* * *$ & $2,96(0,15) * * *$ & $4,59(0,17) * * *$ \\
\hline \multicolumn{7}{|c|}{ Asignación de clases } \\
\hline Prob. (\%) & $16,88(3,41)$ & & $40,63(5,29)$ & & $28,93(4,98)$ & $13,56(3,60)$ \\
\hline Constante & & & $0,88(0,27)$ & $* * *$ & $0,54(0,29) * *$ & $-0,22(0,36)$ \\
\hline
\end{tabular}

Nota: Nivel de significatividad al $* * 5 \%,{ }^{* * * 1 \%}$.

Fuente: Elaboración propia

\subsection{Diseño de instrumentos económicos}

Comprender cómo se distribuye la demanda social por medidas de gestión sostenible de la naturaleza y cómo las preferencias dependen de variables sociodemográficas, de actitud y relacionales es clave para el éxito de las acciones públicas en relación con la gestión de los espacios naturales. Sin embargo, ello no es suficiente si no se discuten sus implicaciones políticas. En otras palabras, ¿cómo pueden los resultados obtenidos ayudar a los gestores públicos a mejorar la gestión de estos espacios?

La amplia gama de instrumentos económicos para apoyar la conservación de la naturaleza cubre tarifas de usuarios, pagos por servicios ecosistémicos, impuestos, subsidios, esquemas ambientales, certificaciones, etc. [Rode et al. (2016)]. Los factores determinantes de la DAP se pueden aplicar al diseño de tales instrumentos económicos. Así, se propusieron dos instrumentos diferentes, un impuesto ambiental y tarifas de usuario.

El impuesto ambiental se puede definir como un impuesto directo, cuya recaudación se utilizará para apoyar la conservación del ENP. La tasa impositiva se calculó a partir de los efectos marginales de la renta estimados por el modelo Tobit, de acuerdo a los tramos establecidos según las clases de renta analizadas. El Cuadro 3 muestra las tasas impositivas propuestas y la cantidad máxima de impuesto ambiental que pagarían los hogares en cada tramo de renta.

Cuadro 3. Impuesto ambiental propuesto para apoyar la gestión sostenible del ENP

\begin{tabular}{|c|c|c|c|c|c|}
\hline \multicolumn{2}{|c|}{ Clúster de renta } & \multirow{2}{*}{$\begin{array}{l}\text { Tramos de renta } \\
(€ / \text { hogar/año) } \\
<12.600 €\end{array}$} & \multirow{2}{*}{$\begin{array}{l}\text { Efecto marginal } \\
2,23 \cdot 10^{-4} * *\end{array}$} & \multirow{2}{*}{ Tasa impositiva (\%) } & \multirow{2}{*}{$\begin{array}{c}\begin{array}{c}\text { Impuesto ambiental } \\
(€ / \text { hogar/año })\end{array} \\
2,81\end{array}$} \\
\hline Clase 1 & Mediana & & & & \\
\hline & Percentil 99 & $12.601 €-22.400 €$ & $2,41 \cdot 10^{-4} * *$ & 0,024 & 5,17 \\
\hline \multirow[t]{3}{*}{ Clase 2} & Mediana & $22.401 €-35.000 €$ & $2,53 \cdot 10^{-4} * *$ & 0,025 & 8,36 \\
\hline & Percentil 99 & $35.001 €-56.000 €$ & $2,66 \cdot 10^{-4} * *$ & 0,027 & 13,94 \\
\hline & & $>56.001 €$ & $2,81 \cdot 10^{-4} * *$ & 0,028 & \\
\hline
\end{tabular}

Nota: Nivel de significatividad al **5\%.

Fuente: Elaboración propia 
El segundo instrumento propuesto fue una tarifa de usuario aplicable a quienes visiten o realicen actividades en el ENP. En este sentido, se asume que cuanto mayor es el comportamiento pro-ambiental de los individuos, mayor es su compromiso ambiental y, por tanto, están dispuestos a pagar más. De manera similar a la propuesta de impuesto ambiental, esta tarifa se estimó a partir de los resultados del modelo Tobit, combinando los efectos marginales del compromiso ambiental con los resultados del análisis de clases latentes. En el Cuadro 4 se muestra las tarifas de usuario propuestas, estimadas por hogar y persona, que podrían oscilar entre aproximadamente 2 y $3,5 € /$ persona/visita.

Cuadro 4. Tarifas de usuario propuestas para para apoyar la gestión sostenible del ENP

\begin{tabular}{lrcrccc}
\hline $\begin{array}{l}\text { Clúster de } \\
\text { compromiso ambiental Afectivo }\end{array}$ & $\begin{array}{r}\text { ICA- Efecto marginal } \\
\text { Clase } 1\end{array}$ & $\begin{array}{c}\text { ICA- } \\
\text { Real }\end{array}$ & Efecto marginal & $\begin{array}{c}\text { Tarifa } \\
(€ / \text { hogar) }\end{array}$ & $\begin{array}{c}\text { Tarifa } \\
(€ / \text { persona) }\end{array}$ \\
\hline Clase 2 & 3,72 & $5,21 * * *$ & 1,83 & $1,30 *$ & 6,50 & 2,08 \\
Clase 3 & 4,88 & $7,46 * * *$ & 1,28 & $1,86 * *$ & 9,31 & 2,98 \\
Clase 4 & 4,89 & $8,20 * * *$ & 2,96 & $2,04 * *$ & 10,23 & 3,27 \\
\hline
\end{tabular}

Nota: Nivel de significatividad al $* 10 \%, * * 5 \%, * * * 1 \%$.

Fuente: Elaboración propia

Se espera que la implementación de estos instrumentos económicos proporcione, en promedio, ingresos financieros de aproximadamente $443.000 €$ /año en el caso del impuesto ambiental y alrededor de 520.000

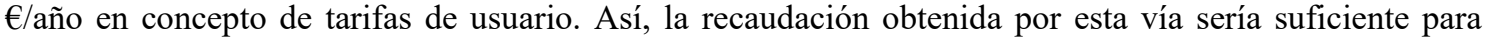
soportar los costes de conservación presupuestados, alrededor de $400.000 €$ /año.

\section{Conclusiones}

La valoración económica de los beneficios que proporcionan los espacios naturales puede no ser suficiente para asegurar su conservación. También es necesario comprender cómo se distribuyen en la sociedad e identificar los factores sociales que determinan su valor. La heterogeneidad en las preferencias sobre medidas de gestión sostenible para un espacio Red Natura 2000 de la Región de Murcia revela que la renta y el compromiso medioambiental son los principales factores que determinan su demanda. Estos resultados sirven de guía a los responsables públicos en el diseño de políticas ambientales socialmente aceptables para apoyar la conservación de estos espacios. Instrumentos económicos, como impuestos ambientales directos y tarifas a los usuarios, han sido propuestos utilizando los resultados de la heterogeneidad de preferencias. Estos instrumentos contribuyen al apoyo financiero de la implementación de medidas de gestión sostenible que gocen de aceptación pública. Se espera que esta investigación contribuya a la comprensión de la demanda social por la conservación sostenible de la naturaleza y a la inclusión de la heterogeneidad de preferencias en el diseño de instrumentos económicos para ayudar a la formulación de políticas.

\section{Agradecimientos}

Este trabajo se ha realizado en el marco del proyecto 20912/PI/18 financiado por la "Fundación SénecaAgencia de Ciencia y Tecnología de la Región de Murcia”. José A. Zabala y José A. Albaladejo-García agradecen la ayuda recibida del MEFP (FPU16/03473 | FPU 16/03562).

\section{Bibliografía}

Ives, C. D. y Kendal, D. (2014). "The role of social values in the management of ecological systems". Journal of Environmental Management, 144: 67-72.

Maloney, M. P., Ward, M. P. y Braucht, G. N. (1975). “A revised scale for the measurement of ecological attitudes and knowledge". American Psychologist, 30(7): 787-790.

Rode, J., Wittmer, H., Emerton, L. y Schröter-Schlaack, C. (2016). “'Ecosystem service opportunities': A practice-oriented framework for identifying economic instruments to enhance biodiversity and human livelihoods". Journal for Nature Conservation, 33: 35-47. 